\title{
Cosmic ray spectra in planetary atmospheres
}

\author{
M. Buchvarova ${ }^{1}$ and P. Velinov ${ }^{2}$ \\ ${ }^{1}$ Space Research Institute, Bulgarian Academy of Sciences, \\ 6 Moskovska Str., Sofia 1000, Bulgaria \\ email: marusjab@yahoo.com \\ ${ }^{2}$ Solar-Terrestrial Influences Laboratory, Bulgarian Academy of Sciences \\ Acad. G. Bonchev, bl.3, Sofia 1113, Bulgaria
}

\begin{abstract}
Our model generalizes the differential $D(E)$ and integral $D(>E)$ spectra of cosmic rays $(\mathrm{CR})$ during the 11-year solar cycle. The empirical model takes into account galactic (GCR) and anomalous cosmic rays (ACR) heliospheric modulation by four coefficients. The calculated integral spectra in the outer planets are on the basis of mean gradients: for GCR $-3 \% / \mathrm{AU}$ and $7 \% / \mathrm{AU}$ for anomalous protons. The obtained integral proton spectra are compared with experimental data, the CRÈME96 model for the Earth and theoretical results of 2D stochastic model. The proposed analytical model gives practical possibility for investigation of experimental data from measurements of galactic cosmic rays and their anomalous component.
\end{abstract}

Keywords. Cosmic ray spectra, modelling, planet atmospheres

\section{Introduction}

The observed CR spectrum can be distributed into the following five energy intervals (Dorman 1977): I $\left(E=3 \times 10^{6}-10^{11} \mathrm{GeV} / \mathrm{n}\right)$, II $\left(E=3 \times 10^{2}-3 \times 10^{6} \mathrm{GeV} / \mathrm{n}\right)$, III $\left(E=30 \mathrm{MeV} / \mathrm{n}-3 \times 10^{2} \mathrm{GeV} / \mathrm{n}\right), \mathrm{IV}(E=1-30 \mathrm{MeV} / \mathrm{n}), \mathrm{V}(E=10 \mathrm{KeV} / \mathrm{n}-$ $1 \mathrm{MeV} / \mathrm{n}$ ), where $E$ is the kinetic energy of the particles. The modulation above $100 \mathrm{GeV}$ is negligible due to the magnetic field of the heliosphere, and the energy spectrum of cosmic rays is described by power law. Particles with energy below $100 \mathrm{GeV}$ are subject to solar modulation due to the presence of the heliosphere. In this paper it is proposed an empirical model for the calculation of the cosmic ray proton and helium spectra on the basis of balloon and satellite measurements in the energy intervals III and IV.

\section{Cosmic ray differential spectrum}

The empirical model for the differential spectrum (energy range $E$ from about $30 \mathrm{MeV}$ to $100 \mathrm{GeV}$ ) of protons and other groups of cosmic ray nuclei on account of the anomalous cosmic rays (energy range $E$ from $1 \mathrm{MeV}$ to about $30 \mathrm{MeV}$ ) is given by Velinov (2000) and Buchvarova (2006):

$$
\begin{aligned}
D(E)=K(0.939+E)^{-\gamma}\left(1+\frac{\alpha}{E}\right)^{-\beta}\left\{\frac{1}{2}[1+\right. & \tanh (\lambda(E-\mu))]\} \\
& +\frac{x}{E^{y}}\left\{\frac{1}{2}[1-\tanh (\lambda(E-\mu))]\right\}
\end{aligned}
$$

The coefficients $\alpha, \beta, x$ and $y$ are related to modulation levels in corresponding energy intervals. The terms with tanh are smoothing functions (Velinov 2002). The dimensionless parameter $\lambda=100$ is inversely proportional to the length of the smoothing interval between the two addends. The physical meaning of $\mu(\mathrm{GeV})$ is the energy at which 
the differential spectrum of GCR crosses the differential spectrum of ACR (Buchvarova 2006).

Experimental data $\left(E_{i}, D_{i}\right)$ for protons and helium nuclei were gotten from Hillas (1972) for solar cycle 20. The power index $\gamma=2.63$ (Hillas 1972). The normalization constants $K p=14.55671 \mathrm{GeV}^{2.63} /\left({\mathrm{s} . \mathrm{m}^{2}}^{2}\right.$ ster. $\left.\mathrm{MeV}\right)$ for protons and $K \alpha=0.90985 \mathrm{GeV}^{2.63} /$ $\left(\mathrm{s} . \mathrm{m}^{2}\right.$ ster. $\left.\mathrm{MeV} / \mathrm{n}\right)$ for alpha particles are chosen to match the modulated data near to $100 \mathrm{GeV} / \mathrm{n}$, where the modulation effect is negligible. The coefficient values for the protons with these model parameters are: $\alpha=9.848438, \beta=0.881461, x=0.000012, y=$ 1.825847, $\mu=0.043094$ at solar minimum and $\alpha=1.108842, \beta=1.029644, x=0.000189$, $y=1.79179, \mu=0.012681$ at solar maximum. We obtain the following values for alpha particles: $\alpha=0.575525 ; \beta=0.622310, x=0.000193, y=1.641234 ; \mu=0.022171$ at solar minimum and the values $\alpha=1.325028, \beta=1.027078, x=0.000059, y=1.432015$ and $\mu=0.034635$ at solar maximum.

The calculation of coefficients $\alpha, \beta, x, y$ and $\mu$ is performed by Levenberg-Marquardt algorithm (Press et al. 1991), applied to the special case of a least squares. The described programme is realized in algorithmic language $\mathrm{C}^{++}$.

\section{Application of the model to planetary atmospheres}

The solar EUV radiation is weaker than the galactic cosmic ray intensity for Jupiter, Saturn, Uranus and Neptune. This shows the importance of the galactic cosmic rays in the formation of outer-planet ionospheres. The integral spectra of the outer planets are obtained using mean integral gradient of GCR - 3\%/AU (Fuji et al. 1997; Heber et al. 1995 ) and 7\%/AU for anomalous protons (McKibben et al. 1979; Christian et al. 1999) and the obtained values of coefficients from Eq. (2.1) for the Earth. For integration we use the equation:

$$
D(>E)=\int_{E}^{\infty} D(E) d E
$$

$D(E)$ - differential spectrum of galactic and anomalous CR, $D(>E)$ - integral spectrum, expressed by the number of particles per unit solid angle, square centimeter, and second, with total energies at least $E$. Integration is performed by Simpson rule (Press et al. 1991).

In Figure 1 is presented the modelled integral spectrum for protons at minimum and maximum of solar activity for Earth and Jupiter. The data for CR protons by Shopper (1967) are noted with crosses and with full squares for the CREME96 model (CREME96). The computations are compared with satellite data: $\boldsymbol{\Delta}$ - Voyager 2 (5 July 1979 - near to solar maximum) for Jupiter and theoretical results of $2 \mathrm{D}$ stochastic model built from Bobik et al. (2006).

It is seen from Figure 1 that in Bobik's model, the integral spectrum of the Earth almost tally with the Jupiter's integral spectrum at solar minimum. It is due to lower average values of integral radial gradient in this model. Actually at high values of the diffusion coefficient radial gradient has lower values in the inner heliosphere. Above few $\mathrm{GeV}$ the difference between modulated spectra for different planets becomes negligible in Bobik's model. In our model integral spectra are computed in first approximation. We assume mean integral gradient of GCR as 3\%/AU for all rigidities, irrespectively of the distance in the heliosphere or solar activity level.

The Bobik's model with the noted parameters (from Figure 2) reproduces comparatively average level of the solar activity. 

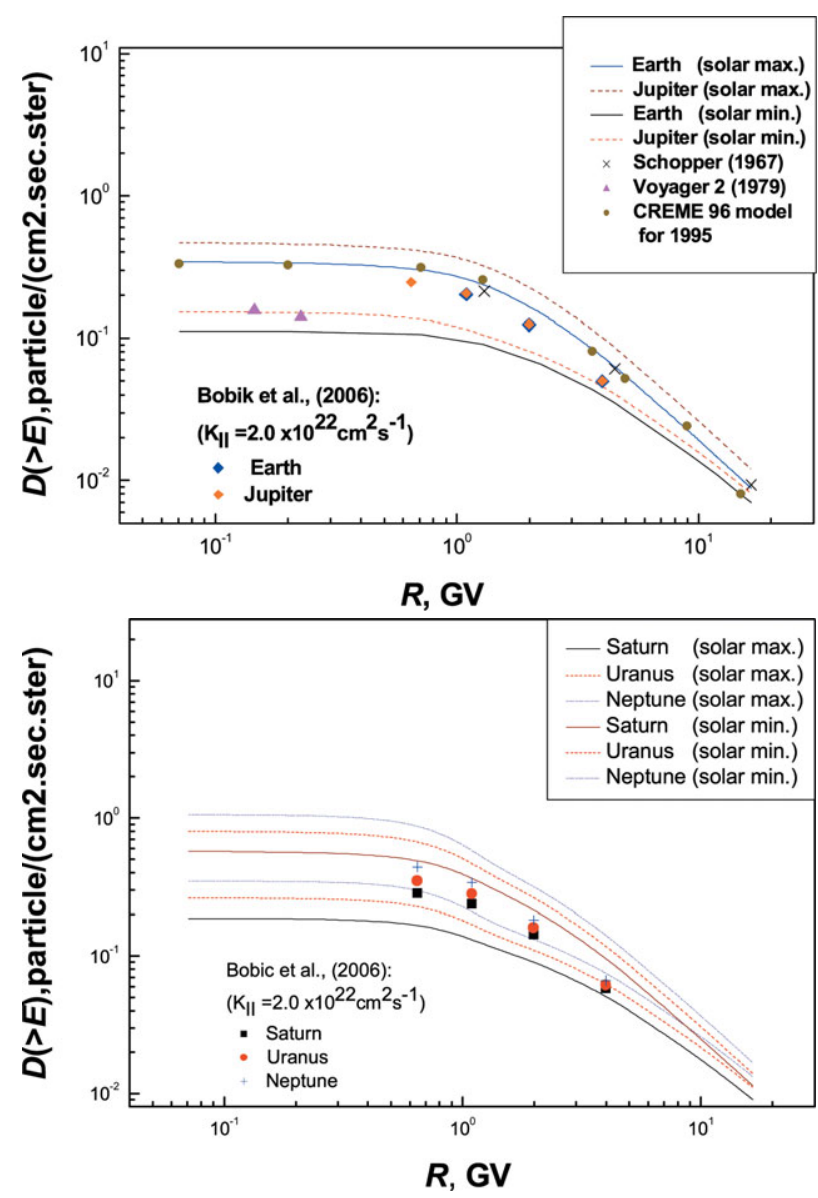

Figure 1. The modelled integral spectra $D(>E)$ of $\mathrm{CR}$ protons for maximum and minimum levels of solar activity for Earth and Jupiter. The computations are compared with the experimental data: $\times$ Schopper (1967) for Earth, $\boldsymbol{\Delta}$ - Voyager2 (5 July 1976 - near to solar minimum) for Jupiter and theoretical results of $2 \mathrm{D}$ stochastic model built from Bobik et al. (2006) and - - CRÈME model (CREME96) for Earth.

Figure 2. The modelled integral spectra $D(>E)$ of $\mathrm{CR}$ protons for maximum and minimum levels of solar activity for Saturn, Uranus and Neptune. The results are compared with computations of Bobik et al. (2006) for 2D transport model with drift.

The calculated integral proton spectra for solar minimum and maximum for Jupiter, Saturn, Uranus and Neptune are show in Figure 2.

\section{References}

Bobik, P. et al. 2006, in: Proc. of 20 ECRS, (Lisbon, Portugal)

Buchvarova, M. 2006, PhD Thesis, Sofia

Christian, E. et al. 1999, in: B. L. Dingus, D. B. Kieda, \& M. H. Salamon (eds.), Proc. of 26 ICRC., Salt Lake City, Utah (USA, New York: American Institute of Physics Press)

CREME96, Cosmic ray ion differential and integral spectra. Available from: http://tecees.esa.int/ProjectSupport/ISO/CREME96.html.

Dorman, L. I. 1977, in: Proc. of 15 ICRC, (Sofia: Bulg. Acad. Sci. Publ. House), p. 405

Fuji, Z. \& McDonald, F. B. 1997, J. Geophys. Res., 102, 24201

Heber, B. et al. 1995, Space Sci. Rev., 72, 391

Hillas, A. M. 1972, Cosmic Rays (Oxford; Pergamon Press)

McKibben, R. B, Pyle, K. R., \& Simpson, J. A. 1979, ApJ. (Letters), 227, L147

Velinov, P. I. Y. 2000, Compt. Rend. Acad. Bulg. Sci., 53, 37.

Press, W. H., Flannery, B. P., Teukolsky, S. A., \& Vetterling, W. T. 1991, Numerical Recipes in $C$ - the Art of Scientific Computing (Cambridge University Press)

Schopper, E. 1967, in: K. Sitte (ed.), Encyclopedia of Physica, v. XL (Berlin: Springer), p. 372 